Detecting early erosive tooth wear using an intraoral scanner system

Michou, Stavroula; Vannahme, Christoph; Ekstrand, Kim R.; Benetti, Ana R.

Published in:

Journal of Dentistry

DOI:

10.1016/j.jdent.2020.103445

Publication date:

2020

Document version

Early version, also known as pre-print

Citation for published version (APA):

Michou, S., Vannahme, C., Ekstrand, K. R., \& Benetti, A. R. (2020). Detecting early erosive tooth wear using an intraoral scanner system. Journal of Dentistry, 100, [103445]. https://doi.org/10.1016/j.jdent.2020.103445 


\section{Detecting early erosive tooth wear using an intraoral scanner system}

Stavroula Michou ${ }^{\mathrm{a}, \mathrm{b}, *}$, Christoph Vannahme ${ }^{\mathrm{b}}, \mathrm{Kim}_{\mathrm{R}}$. Ekstrand ${ }^{\mathrm{a}}$, Ana R. Benetti ${ }^{\mathrm{a}}$

a Department of Odontology, Faculty of Health and Medical Sciences, University of Copenhagen, Copenhagen, Denmark

b 3Shape TRIOS A/S, Copenhagen, Denmark

Publication date: 2020 Aug 1

Document version: Pre-pint accepted manuscript

Citation for published version (APA):

Michou S, Vannahme C, Ekstrand KR, Benetti AR. Detecting early erosive tooth wear using an intraoral scanner system. J Dent. 2020;103445. doi:10.1016/j.jdent.2020.103445 


\title{
Detecting early erosive tooth wear using an intraoral scanner system
}

\author{
Stavroula Michou ${ }^{\mathrm{a}, \mathrm{b}, *}$, Christoph Vannahme ${ }^{\mathrm{b}}$, Kim R. Ekstrand ${ }^{\mathrm{a}}$, Ana R. Benetti ${ }^{\mathrm{a}}$ \\ ${ }^{a}$ Department of Odontology, Faculty of Health and Medical Sciences, University of Copenhagen, \\ Copenhagen, Denmark \\ b 3Shape TRIOS A/S, Copenhagen, Denmark
}

Keywords: Tooth wear, Tooth erosion, Imaging, Three-dimensional, Diagnostic imaging

\begin{abstract}
Objectives: To assess the feasibility of detecting and monitoring early erosive tooth wear using a 3D intraoral scanner (IOS) aided by specific software.
\end{abstract}

Methods: Extracted sound permanent teeth were assembled in two shortened artificial dental arches and scanned at different intervals with an IOS (3Shape TRIOS $® 3$ ) before and after an erosion/abrasion protocol (i.e. $1 \mathrm{~h}$ up to $24 \mathrm{~h}$ immersion in citric acid solution and subsequent brushing). The 3D models obtained at consecutive time points were superimposed with the baseline model using dedicated software (3Shape TRIOS® Patient Monitoring, version 2.1.1.0) and reference surface alignment. Surface profile differences between the baseline 3D model and the respective models from different time points were expressed as tooth substance loss.

Non-parametric tests were used to assess the significance of tooth substance loss at different time points. Spearman's correlation was applied between the tooth substance loss at the end of each erosion/abrasion cycle and the immersion time in acid.

Results: Significant tooth substance loss $(0.08 \mathrm{~mm}, \mathrm{IQR}=0.05)$ was detected by the software after $3 \mathrm{~h}$ of erosive-abrasive challenge $(\mathrm{p}=0.045)$. The overall median loss increased gradually from baseline to $24 \mathrm{~h}$ showing a strong correlation with the immersion time in acid $\left(\mathrm{r}_{\mathrm{s}}=0.971, \mathrm{p}<0.01\right)$.

Conclusions: The use of an IOS aided by specific software showed good performance for early detection and monitoring of tooth wear in vitro and has promising potential for in vivo application. Clinical significance: Detection and monitoring of early erosive tooth wear can be reliably aided by intraoral scanning supported by specific software. The measurement error and uncertainty involved in this method should be taken into consideration when interpreting the tooth substance loss measurements. Furthermore, presuming the difficulty in defining reference surfaces in vivo, clinical validation is needed to determine the system's in vivo performance.

\section{Introduction}

Tooth wear is a multifactorial condition with increasing incidence globally, particularly in Western societies [1]. Early detection and monitoring of tooth wear remains extremely challenging using traditional clinical examination methods $[2,3]$.

Clinical detection and diagnosis of tooth wear is usually based on visual examination of lesion characteristics, sometimes also supported by the acquisition of clinical photographs and study models [3-5]. However, visual examination is subjective even when specific criteria such as the Basic Erosive Wear Examination (BEWE) [6] are adopted, as these have previously shown only moderate reproducibility when employed by general practitioners [7]. Furthermore, visual assessment shows low 
sensitivity; tooth mineral loss is usually only visually perceptible when a significant amount of hard dental tissue is already lost. Another disadvantage of the visual examination is inability to quantify tooth substance loss over time, a significant limitation when the activity and progression rate of the disease needs to be defined in order to decide the patient's treatment plan [3].

A number of methods other than visual examination have been assessed to aid detection and/or quantification of tooth substance loss [2,5]. Numerous in vitro studies have employed techniques including quantitative light-induced fluorescence (QLF) [8-10], profilometry [11-15], and optical coherence tomography (OCT) $[8,16]$. Applying these methods in vivo is difficult; for example, the use of profilometry is time-consuming as it requires obtaining physical models of the dentition. QLF and OCT, although they can potentially be applied directly in the mouth [17], show suboptimal reproducibility in longitudinal studies involving patients [2,3,5,18,19].

The use of IOS has been recently suggested for early detection, quantification, and monitoring of tooth wear based on in vitro [20-22] as well as in vivo studies examining 3D data obtained directly from patients [23] or cast models [24]. Studies focusing on visual detection of early erosive tooth wear demonstrated detection with higher sensitivity using meticulous visual examination (BEWE index) on full-arch digital 3D models compared to traditional visual examination on patients [23,24]. This finding was attributed to the larger magnification and clearer image of the dentition allowed by the digital 3D model compared to the traditional visual examination directly on the patient. Other studies went further by using specific software to align multiple 3D models, from which measurements of profile or volumetric differences were obtained [20-22,25,26].

In order to develop an objective, automated, and reliable in vivo method for early detection and monitoring of tooth wear, the use of an accurate IOS is essential [27,28], in addition to a reliable method for 3D model alignment with minimal error $[20,21,25]$. This study builds upon the existing knowledge derived from in vitro models with single-tooth set-ups [20,24,25], in which the accumulation of errors related to scanning and tooth alignment is relatively small, and approaches the clinical scenario by investigating erosive tooth wear on a set-up designed to simulate the in vivo dentition. Thus, this study aimed to assess the feasibility of an IOS aided by specific software to detect, quantify and monitor erosive tooth wear on study models comprising of multiple and different types of teeth and multiple tooth surfaces.
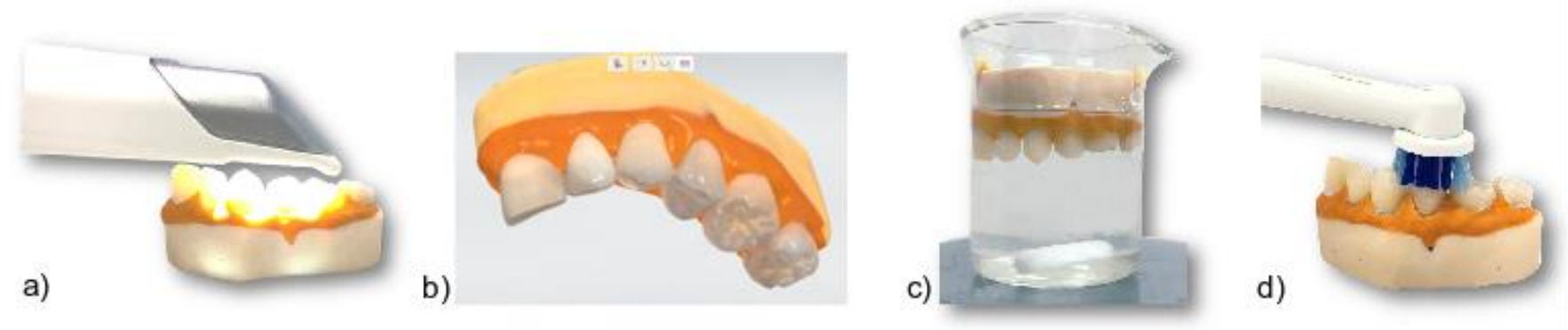

Fig. 1. Experiment workflow followed in the study. Scanning with $\operatorname{IOS}(a)$, obtaining the $3 D$ model (b), immersion in the acid solution for simulated erosion (c), tooth brushing for simulated abrasion $(d)$.

\section{Materials and methods}

\subsection{Specimen preparation}

Twelve $(\mathrm{N}=12)$ sound human teeth (anterior and posterior) without visual surface defects were selected from a pool of extracted teeth from the Department of Odontology of the University of 
Copenhagen. All teeth were extracted for therapeutic reasons by dental practitioners from anonymous donors and collected as leftover biological material; therefore, no notification to the National Ethical Committee was required. The teeth were stored in chloramine T trihydrate aqueous solution $(0.5 \%$ w/v) until use.

After selection, the teeth were mounted on a dimensionally stable silicone impression material in an artificial semi-arch set-up to simulate an in vivo model. Two dental semi-arches of 6 teeth were fabricated. Each semi-arch included a sequence of teeth from central incisor to second molar. Composite restorations (Filtek Z250 3M ESPE, 3M Deutschland $\mathrm{GmbH}$ ) covering two or more dental surfaces were made on some teeth using selective enamel etching and bonding (3M $\mathrm{M}^{\mathrm{TM}}$ Scotchbond ${ }^{\mathrm{TM}}$ Universal Etchant and Adhesive 3M ESPE, 3M Deutschland $\mathrm{GmbH}$ ). Composite restorations in the first semi-arch were placed on the central (class III, distal) and lateral incisors (class IV, mesial), premolar (class II, occluso-distal), and second molar (class II, mesio-occluso-palatal). In the second semi-arch, composite restorations were placed on the central (class IV, mesial) and lateral incisors (class IV, mesial), canine (class III, distal), first (class II, distal) and second molars (class II, mesiooccluso-palatal). The restorations were used to establish reference surfaces for the 3D model alignments, as the resin composite is relatively resistant to erosive-abrasive challenge [29].

The teeth were stored for 1-7 days in phosphate-buffered saline solution $(\mathrm{NaCl} 137 \mathrm{mmol} / \mathrm{L}, \mathrm{KCl} 2.7$ $\mathrm{mmol} / \mathrm{L}$, Na2HPO4 $10 \mathrm{mmol} / \mathrm{L}, \mathrm{KH} 2 \mathrm{PO} 41.8 \mathrm{mmol} / \mathrm{L}$ ), $\mathrm{pH}$, at $37{ }^{\circ} \mathrm{C}$ until the experiment started.

\subsection{Erosive tooth wear challenge \& 3D Scanning}

All teeth were scanned twice at baseline (baseline 3D models 1 and 2) with the IOS (TRIOS 3®, 3Shape TRIOS A/S, Denmark) according to the manufacturer's suggested scan strategy.

Afterwards, the teeth were immersed in $1 \%$ citric acid solution $(\mathrm{pH} 2.7)$ under continuous agitation (100 rpm) and for each designated time point $(1,2,3,4,5$, and $6 \mathrm{~h})$ the teeth were removed from the solution, rinsed with deionized water (20 s), dried with oil-free compressed air (5 s) and scanned to evaluate erosion. The teeth were subsequently brushed $(2 \mathrm{~min}$ ) with an electrical toothbrush (Oral B Vitality Cross Action, Braun) without toothpaste and then scanned again to evaluate abrasion (Fig. 1). Thus, 3D models were obtained at baseline, immediately after removal from the acid solution (erosion) and finally immediately after toothbrushing (abrasion).

Subsequently, only the first semi-arch was further assessed every $6 \mathrm{~h}$ up to $24 \mathrm{~h}$. In summary, 3D models of the first semi-arch were obtained at baseline and 9 subsequent time points $(1,2,3,4,5,6$, 12,18 and $24 \mathrm{~h}$ ); for the second semi-arch, at baseline and 6 subsequent time points $(1,2,3,4,5$ and 6 h). If storage for some hours between the different experimental cycles was needed, the models were stored in deionized water.

\subsection{D model assessment}

The 3D models obtained at the different time points were superimposed with the baseline model no. 1 using specific software (3Shape TRIOS® Patient Monitoring, version 2.1.1.0, 3Shape A/S, Denmark), not commercially available when the study was conducted. Reference alignment was used for superimposition of the models as this method has been shown to result in lower alignment errors and truer measurements [25] compared to other types of alignment. For the reference alignment, the surfaces of the composite restorations were marked manually on each individual 3D model as regions of interest using a brush tool (Fig. 2). The marked surfaces were then used by the software as stable references to align the consecutive 3D models. Thus, the unrestored tooth surfaces, subject to changes due to the tooth wear challenge, were not considered for alignment. 


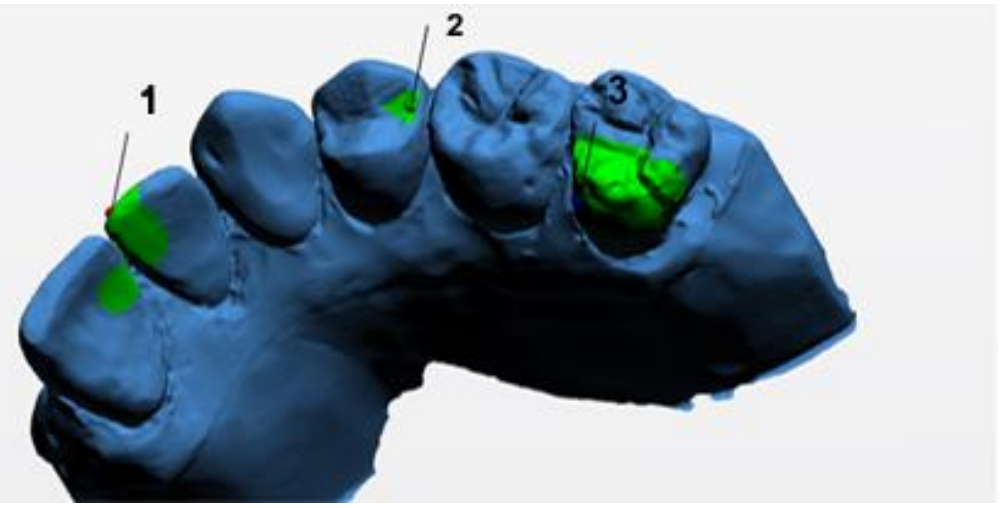

Fig. 2. Illustration of the reference surfaces used for $3 D$ model alignment. The surfaces of composite restorations (green areas) are marked on the model using a brush tool for subsequent reference model alignment.

Following alignment, a colour map tool enabled differences between the baseline model and subsequent models obtained at the different time points to be visualised (Fig. 3). The threshold above which surface changes were visible on the colour map was set in the software as $0.05 \mathrm{~mm}$. The first visual changes above the predefined threshold were noted for each semi-arch.
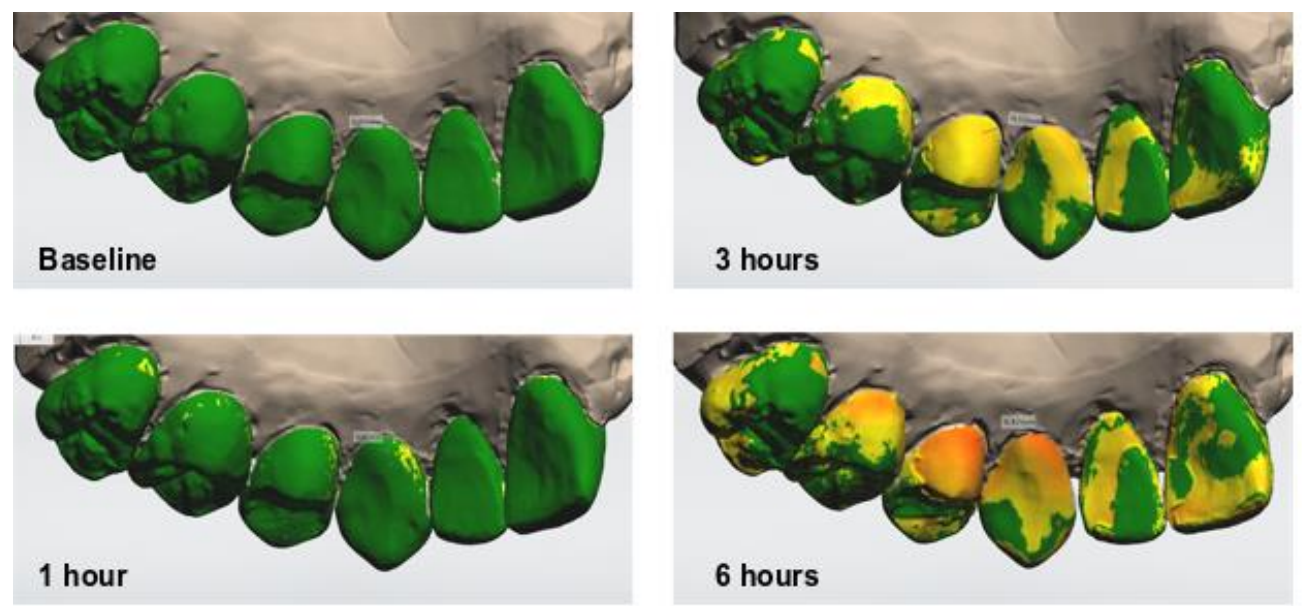

$0.80 \mathrm{~mm}$
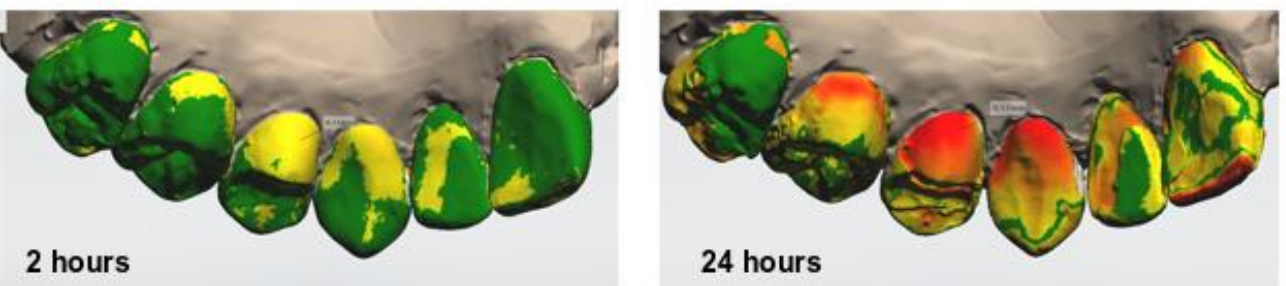

$0.05 \mathrm{~mm}$

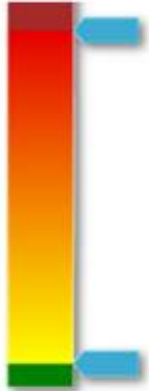

Fig. 3. Representative colour map images showing differences compared to the baseline $3 D$ model at different time points according to the colour scale. Differences below $0.05 \mathrm{~mm}$ (software threshold) are shown as green.

Surface profile differences between baseline and the subsequent 3D models were measured on each tooth using a cross-section tool available in the software (Fig. 4). Using this tool, profile difference measurements as small as $0.01 \mathrm{~mm}$ could be taken on the 3D models to quantify tooth substance loss. Eight measurements, 4 on the palatal and 4 on the buccal surfaces of each tooth, were obtained. Measurements on each tooth were taken on the same positions at all time points by maintaining the position of the cross-sections over time.

\subsection{Measurement error and uncertainty}

The accuracy of the IOS 3Shape TRIOS® 3 has been investigated previously. In a well-controlled study [28], based on a model with equivalent extension to the dental semi-arches investigated in the present study, this scanner's trueness and precision were reported to be $0.025 \mathrm{~mm}$ (standard deviation 
$(\mathrm{SD})=0.005)$ and $0.003 \mathrm{~mm}(\mathrm{SD}=0.002)$, respectively. Based on the reported trueness, we considered the method's measurement error to be $0.025 \mathrm{~mm}$.

In this study, in order to assess the uncertainty associated with the reference 3D model alignment alone, or in combination with 3D scanning, ten repetitions of each of the following assessments were conducted:

1. Alignment and surface profile difference measurements between two identical copies of the same baseline 3D model (i.e. baseline model 1 with itself);

2. Alignment and surface profile difference measurements between two non-identical baseline 3D models, obtained from the same dental semi-arch (i.e. baseline models 1 and 2).

The composite fillings were again used as reference for the model alignments and the surface profile differences were measured at cross sections of the 3D models as conducted in the main experiment, i.e. eight measurements per tooth. Mean surface profile differences and SDs were calculated for each assessment and used for measurement uncertainty calculations.

Taking into account all the individual measurement uncertainties associated with the scanning, model alignment and surface profile measurements deriving both from the study of Mennito et al. [28] and the present study, a combined measurement uncertainty was calculated [30].
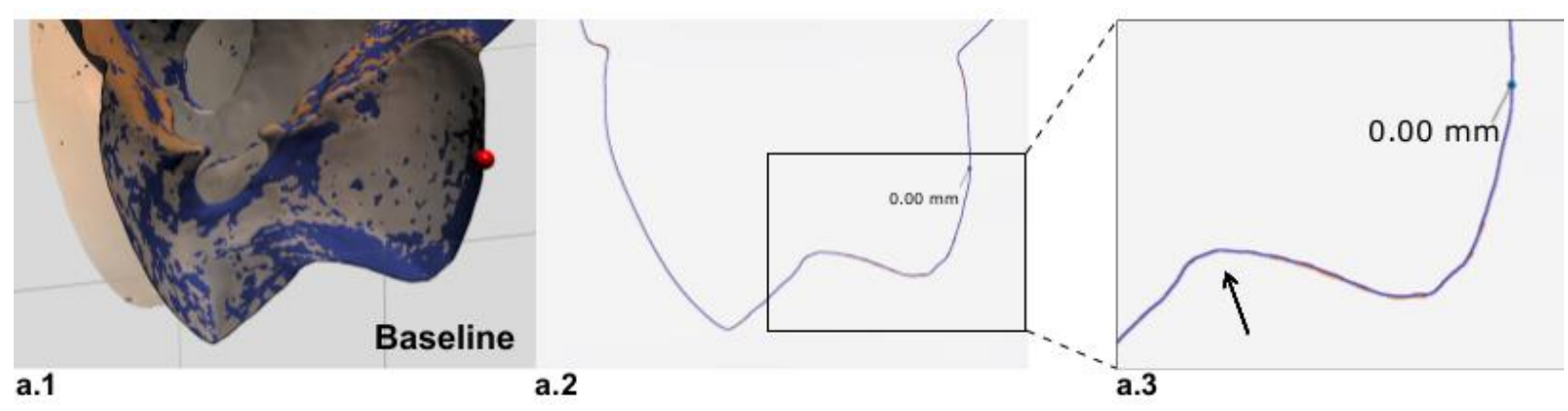

a.1

a.2
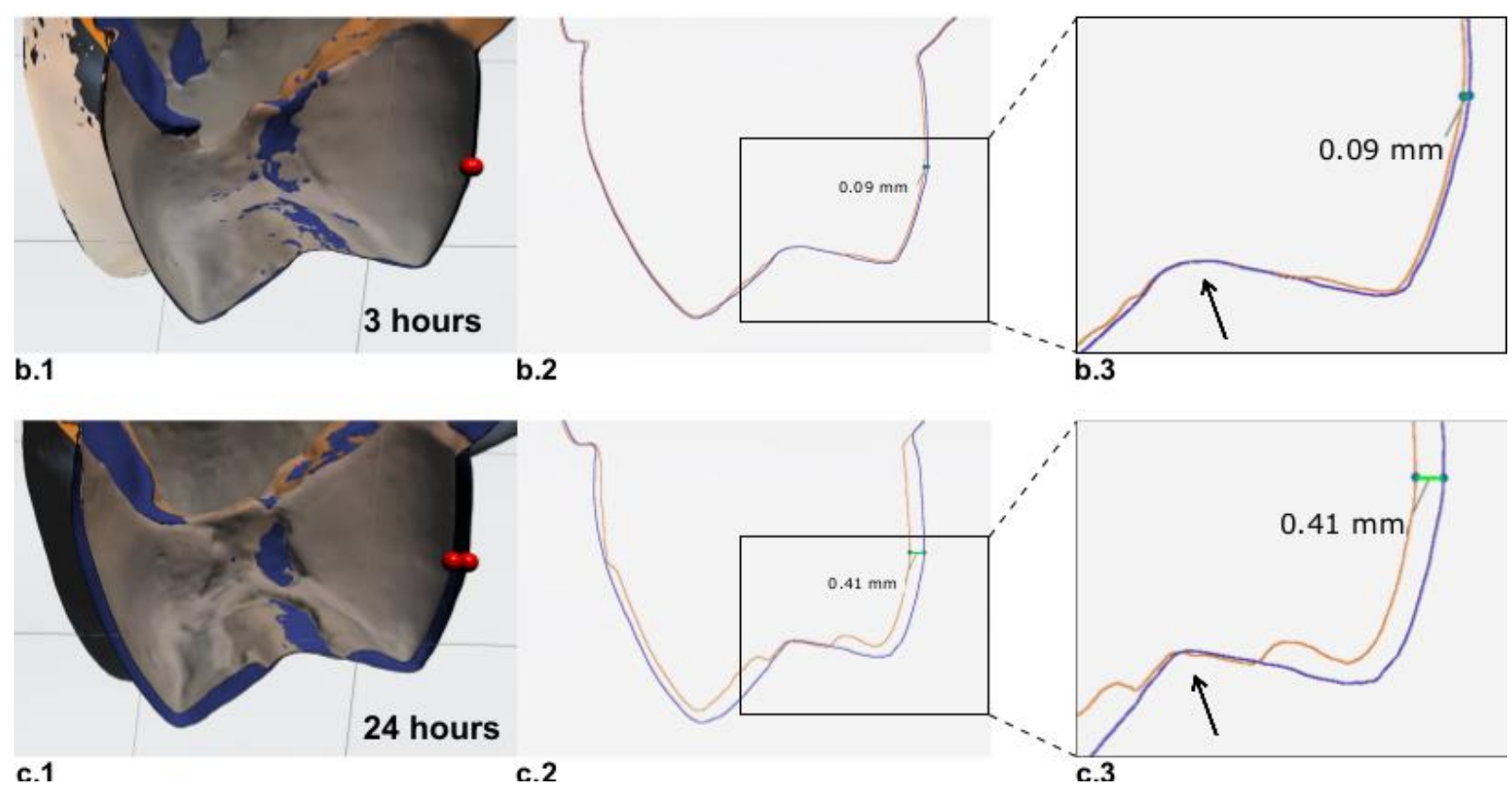

Fig. 4. Representative cross-sections of $3 D$ models at different time points superimposed with the baseline model. Cross-sectioned, aligned 3D models (a.1, b.1, c.1), simple linear representation of cross-sections used for profile difference measurements $(a .2, b .2, c .2)$ and their magnifications $(a .3, b .3, c .3)$ are provided. The composite restoration used for reference model alignment is marked with an arrow. 


\subsection{Statistics}

Median surface profile difference and interquartile range (IQR) were calculated for each tooth, then for each model and subsequently for both models as a function of time. Data were analysed using SPSS software (IBM SPSS Statistics 26 for Windows, Version 26.0; IBM Corp, New York, US). ShapiroWilk test was used to assess the normality of the data as well as normal Q-Q plots and histograms. Not all sub-groups were normally distributed, therefore Friedman test followed by Dunn's post-hoc were used to assess the significance of tooth substance loss between different time points. Spearman's correlation coefficient $\left(\mathrm{r}_{\mathrm{s}}\right)$ was calculated between the median surface profile difference $(\mathrm{mm})$ after toothbrushing and the hours of immersion in acid. Level of significance was set at $a=0.05$.

\section{Results}

\subsection{Tooth substance loss}

Visual differences above $0.05 \mathrm{~mm}$ (software threshold) on the colour map were observed from $1 \mathrm{~h}$ of immersion in acid (Fig. 3).

Median profile difference (IQR) [mm]

\begin{tabular}{cc|c|c|c|c|c}
\hline & \multicolumn{3}{c|}{ Erosion } & \multicolumn{3}{|c}{ Abrasion } \\
Time [h] & Semi-arch 1 & Semi-arch 2 & Median & Semi-arch 1 & Semi-arch 2 & Median \\
\hline (Baseline) & $0.00(0.01)$ & $0.01(0.01)$ & $0.01(0.01)$ & & & \\
1 & $0.01(0.02)$ & $0.01(0.04)$ & $0.01(0.02)$ & $0.02(0.01)$ & $0.02(0.03)$ & $0.02(0.01)$ \\
2 & $0.05(0.03)$ & $0.02(0.05)$ & $0.04(0.03)$ & $0.06(0.02)$ & $0.04(0.02)$ & $0.05(0.02)$ \\
3 & $0.07(0.01)$ & $0.08(0.05)^{*}$ & $0.07(0.02)$ & $0.08(0.02)$ & $0.08(0.06)^{*}$ & $0.08(0.02)$ \\
4 & $0.10(0.02)^{*}$ & $0.12(0.02)^{*}$ & $0.11(0.02)^{*}$ & $0.12(0.02)^{*}$ & $0.10(0.02)^{*}$ & $0.10(0.02)^{*}$ \\
5 & $0.14(0.03)^{*}$ & $0.14(0.08)^{*}$ & $0.14(0.04)^{*}$ & $0.14(0.03)^{*}$ & $0.16(0.06)^{*}$ & $0.15(0.03)^{*}$ \\
6 & $0.16(0.03)^{*}$ & $0.16(0.08)^{*}$ & $0.16(0.03)^{*}$ & $0.17(0.02)^{*}$ & $0.18(0.05)^{*}$ & $0.18(0.03)^{*}$ \\
12 & $0.19(0.03)^{*}$ & & & $0.23(0.04)^{*}$ & & \\
18 & $0.24(0.04)^{*}$ & & & $0.28(0.04)^{*}$ & & \\
24 & $0.29(0.05)^{*}$ & & & $0.32(0.04)^{*}$ & \\
\hline
\end{tabular}

Table 1. Median surface profile difference [mm] at different points in time [h] after removal from the acid solution (erosion) and after tooth brushing (abrasion). Interquartile range (IQR) is provided in parentheses. Median values for each dental semi-arch and the overall median values are presented separately. The significant measurements, when compared to baseline values, are marked with *.

Table 1 and Fig. 5 show the surface profile differences obtained using the software's cross-section tool. The overall median surface profile difference between the baseline models and those obtained following erosive-abrasive challenge gradually increased from $0.01 \mathrm{~mm}$ ( $\mathrm{IQR}=0.02$ ) to $0.18 \mathrm{~mm}$ $(\mathrm{IQR}=0.03$ ) after $6 \mathrm{~h}$ and, for the first dental semi-arch, up to $0.32 \mathrm{~mm}(\mathrm{IQR}=0.04)$ after $24 \mathrm{~h}$.

Dunn's test showed that the first significant surface profile difference considering the median from both models was $0.11 \mathrm{~mm}$ ( $\mathrm{IQR}=0.02, \mathrm{p}=0.038$ ), which was observed after $4 \mathrm{~h}$ immersion in the acid. When assessing each model independently, the first significant loss $(0.10 \mathrm{~mm}, \mathrm{IQR}=0.02, \mathrm{p}=0.038)$ 
was observed for the first dental semi-arch after $4 \mathrm{~h}$ in the acid; for the second dental semi-arch, significant loss $(0.08 \mathrm{~mm}, \mathrm{IQR}=0.05, \mathrm{p}=0.038)$ was noticed already after $3 \mathrm{~h}$.

A strong correlation was found between the time of immersion in acid and tooth substance loss at the end of each erosion-abrasion cycle $\left(\mathrm{r}_{\mathrm{s}}=0.971, \mathrm{p}<0.01\right)$.

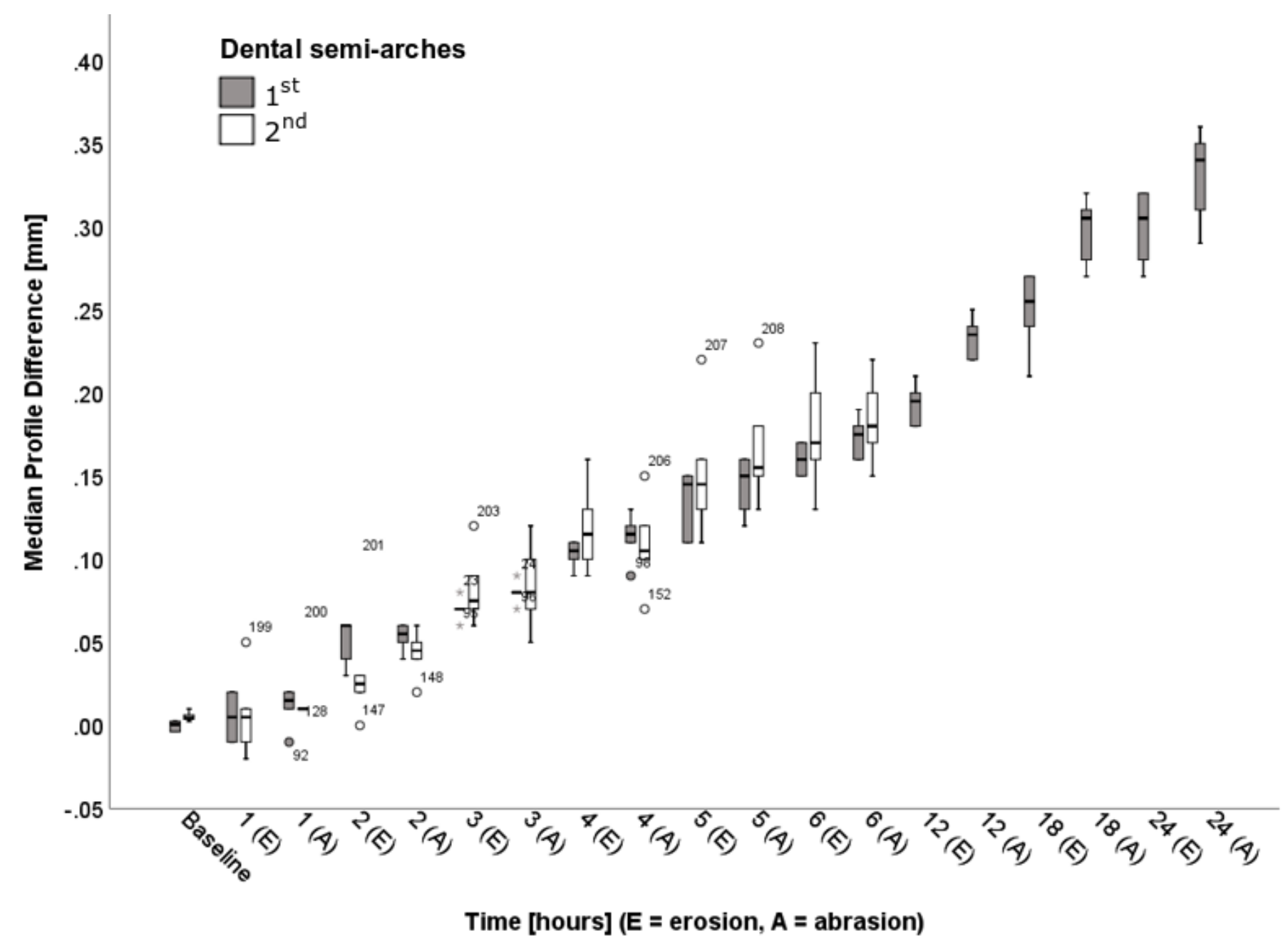

Fig. 5. Clustered boxplot of tooth substance loss as a function of time. The boxplot shows the median surface profile difference [mm] at different time points $(1-24$ h) for each dental semi-arch separately. The measurements obtained immediately after removal from the acid solution are marked with $E$ (erosion) and the measurements obtained after tooth brushing with A (abrasion).

\subsection{Measurement uncertainty}

Mean surface profile difference when assessing the alignment of identical 3D models is $0.00 \mathrm{~mm}$ $(\mathrm{SD}=0.00)$; this value after alignment of non-identical 3D models of the same dental semi-arch was $0.00 \mathrm{~mm}(\mathrm{SD}=0.01 \mathrm{~mm})$.

Taking into account all the individual measurement uncertainties associated with scanning, model alignment, and surface profile measurements, deriving both from Mennito et al. [28] and from the current study, a combined measurement uncertainty was calculated to be $\pm 0.01 \mathrm{~mm}$ at a level of confidence of $95 \%$.

\section{Discussion}

This study shows promising results for the capability of IOS and dedicated software for early detection and monitoring of tooth wear. The relatively low measurement error $(0.025 \mathrm{~mm}$ [28]) and uncertainty 
$( \pm 0.01 \mathrm{~mm})$ combined with the strong correlation between the median tooth substance loss and the time of immersion in acid indicate that this IOS system can reliably detect and monitor progressive tooth substance loss. By combining measurement error and uncertainty, surface profile differences $>0.035 \mathrm{~mm}$ represent true tooth substance loss.

Taking into account that the accuracy of a long span 3D model, as featured in this study, is lower compared to single tooth models, we can foresee even smaller measurement error and uncertainty if the same method is tested on single tooth models [20,21,25,28]. Accordingly, larger measurement error and uncertainty are expected in full-arch 3D models [28]. Furthermore, it is worth mentioning that no powder was needed for scanning with the IOS system used, a notable point as a previous study reported part of the measurement error had resulted from the presence of powder on the surfaces of interest [21].

Composite restorations were used as references for 3D model alignment to minimize the measurement uncertainty. Reference alignment, as supported by previous research, demonstrated reduced errors in alignment and measurements when compared to best fit alignment, the latter available in most software tools [25]. In a clinical scenario, relatively stable surfaces, such as existing restorations or dental surfaces that are expected to be less affected by tooth wear, should preferably be chosen as references for more accurate model alignment. This could be for example the buccal or lingual tooth surfaces in case of erosive wear due to intrinsic or extrinsic factors, respectively. If no stable reference surfaces can be defined, the best fit alignment can be applied as a first step to determine which surfaces show the least differences compared to the baseline model; subsequently, these same surfaces can be used to conduct the reference alignment. Additionally, single tooth alignment, an alternative that could potentially lead to even smaller measurement uncertainty, involves defining reference surfaces on every tooth, something that requires additional time or might not always be possible due to the lack of reference on every tooth. Therefore, alignment and comparison of the complete model were chosen in the current study to more closely mimic future clinical use, as fewer reference surfaces - only on selected teeth - are needed for the model alignment. Model alignment is particularly advantageous to obtain measurements on teeth that may be affected by tooth wear on all surfaces and do not have an easily identifiable reference surface.

In contrast to previous similar studies [20,21,25], which used single-tooth models or enamel samples to investigate $3 \mathrm{D}$ systems for detection and monitoring of tooth wear, the current study used dental semi-arches as study models, more reflective of in vivo presentation. The semi-arches included full crowns of multiple teeth where more than one tooth surface was examined, unlike previous studies which assessed individual surfaces of teeth, usually polished to achieve a flat examination plane and often surrounded by a reference plane unaffected by the erosion-abrasion challenge [8,11-17]. The latter set-up could more easily allow comparison of the results using other equipment such as surface profilers or optical coherence tomography (OCT) scanners. The possibility of including a surface profiler or OCT scanner in the current study as comparison methods [8,11-17] was contemplated. However, due to the limited scan range of such methods when it comes to the assessment of comparably large three-dimensional models and considering the available data supporting the validity of the IOS system employed in this study [27,28], no comparison method was adopted.

The IQR of the profile difference increased with immersion time in acid from $0.01 \mathrm{~mm}$ to 0.08 in the second model. The increase in the measurement range can be attributed to the fact that multiple surfaces on teeth from different people were assessed. It is known that the enamel composition and thus the tooth wear rate varies from person to person or even from one tooth surface to another [3], which may explain some of the outliers within the measurements. For example, the outliers no. 199- 
208 in the second dental semi-arch (Fig. 5) correspond to the same tooth, which was possibly more susceptible to tooth wear than the rest of the sample.

Finally, it was difficult to directly compare the absolute profile difference measurements with findings from previous studies due to methodological differences, e.g. types of measurement devices, differences in erosion-abrasion protocols and measurement criteria. Nevertheless, our findings open for the possibility of further work focusing on the reduction of measurement error and automated calculation of tooth substance loss. Additionally, longitudinal clinical trials to assess the system's in vivo performance in detecting and monitoring early tooth substance loss would be of great value.

\section{Conclusion}

Tooth substance loss due to erosive tooth wear was detected by the IOS system at very initial stages, with the smallest significant loss for the samples at $0.11 \mathrm{~mm}$. Furthermore, increasing tooth substance loss after consecutive erosion-abrasion cycles was quantified showing significant correlation with the exposure time in the acid. Therefore, the use of the IOS aided by specific software for early detection and monitoring of tooth wear is encouraging and can potentially be applied in vivo.

\section{Authorship contribution statement}

Stavroula Michou: Conceptualization, Methodology, Software, Validation, Formal analysis, Investigation, Writing - original draft, Visualization, Project administration. Christoph Vannahme: Conceptualization, Resources, Software, Writing - original draft, Visualization, Supervision, Funding acquisition. Kim R. Ekstrand: Conceptualization, Writing - review \& editing, Supervision, Funding acquisition. Ana R. Benetti: Conceptualization, Methodology, Resources, Validation, Formal analysis, Writing - original draft, Visualization, Supervision, Funding acquisition.

\section{Declaration of Competing Interest}

The authors declare the following financial interests/personal relationships which may be considered as potential competing interests: The current study was funded by Innovation Fund Denmark (Grand Solution project 13-2014-1). At the time that the experimental part of the study and data analysis was conducted (December 2018 - January 2019), the first author (S. Michou) was employed at the University of Copenhagen. At the moment and while writing the submitted manuscript, S. Michou is a $\mathrm{PhD}$ student at the University of Copenhagen, Denmark and is employed at 3Shape TRIOS A/S. Her salary is partially covered by Innovation fund Denmark (grant no. 8053-00005B) based on the foundation's guidelines and according to an industrial $\mathrm{PhD}$ agreement between the industrial partner 3Shape TRIOS A/S and the University of Copenhagen. Furthermore, the co-author C. Vannahme is employed at 3Shape TRIOS A/S. The other co-authors AR. Benetti, KR. Ekstrand declare no conflicts of interest.

\section{Acknowledgement}

Authors acknowledge the laboratory technician Liselotte Larsen for assistance with the preparation of materials needed for the experiments, the TRIOS Patient Monitoring development team at 3Shape for technical support and Innovation Fund Denmark for financial support (Grand Solution project 132014-1). 


\section{References}

[1] N. Schlueter, B. Luka, Erosive tooth wear - a review on global prevalence and on its prevalence in risk groups, Br. Dent. J. 224 (2018) 364-370, https://doi.org/10.1038/sj.bdj.2018.167.

[2] N. Schlueter, A. Hara, R.P. Shellis, C. Ganss, Methods for the measurement and characterization of erosion in enamel and dentine, Caries Res. 45 (2011) 13-23, https://doi.org/10.1159/000326819.

[3] C. Ganss, A. Lussi, Diagnosis of erosive tooth wear, Monogr. Oral Sci. 25 (2014) 22-31, https://doi.org/10.1159/000359935.

[4] C.H. Wilder-Smith, A. Materna, L. Martig, A. Lussi, Longitudinal study of gastroesophageal reflux and erosive tooth wear, BMC Gastroenterol. 17 (2017) 1-7, https://doi.org/10.1186/s12876-017-06701 .

[5] T. Attin, F.J. Wegehaupt, Methods for assessment of dental erosion, Erosive tooth wear from diagnosis to read therapy, (2012), pp. 123-142, https://doi.org/10.1159/000360355.

[6] D. Bartlett, C. Ganss, A. Lussi, Basic erosive wear examination (BEWE): a new scoring system for scientific and clinical needs, Clin. Oral Investig. 12 (2008) 65-68, https://doi.org/10.1007/s00784-0070181-5.

[7] B. Dixon, M.O. Sharif, F. Ahmed, A.B. Smith, D. Seymour, P.A. Brunton, Evaluation of the basic erosive wear examination (BEWE) for use in general dental practice, Br. Dent. J. 213 (2012) pageE4, https://doi.org/10.1038/sj.bdj.2012.670.

[8] H.P. Chew, C.M. Zakian, I.A. Pretty, R.P. Ellwood, Measuring initial enamel erosion with quantitative light-induced fluorescence and optical coherence tomography: an in vitro validation study, Caries Res. 48 (2014) 254-262, https://doi.org/10.1159/000354411.

[9] V. Elton, L. Cooper, S.M. Higham, N. Pender, Validation of enamel erosion in vitro, J. Dent. 37 (2009) 336-341, https://doi.org/10.1016/j.jdent.2009.01.006.

[10] I.A. Pretty, W.M. Edgar, S.M. Higham, The validation of quantitative light-induced fluorescence to quantify acid erosion of human enamel, Arch. Oral Biol. 49 (2004) 285-294, https://doi.org/10.1016/j.archoralbio.2003.11.008.

[11] J.M. Rodriguez, R.S. Austin, D.W. Bartlett, A method to evaluate profilometric tooth wear measurements, Dent. Mater. 28 (2012) 245-251, https://doi.org/10.1016/j.dental.2011.10.002.

[12] J.M. Rodriguez, R.S. Austin, D.W. Bartlett, In vivo measurements of tooth wear over 12 months, Caries Res. 46 (2012) 9-15, https://doi.org/10.1159/000334786.

[13] S. Ranjitkar, A. Turan, C. Mann, G.A. Gully, M. Marsman, S. Edwards, J.A. Kaidonis, C. Hall, D. Lekkas, P. Wetselaar, A.H. Brook, F. Lobbezoo, G.C. Townsend, Surfacesensitive microwear texture analysis of attrition and erosion, J. Dent. Res. 96 (2017) 300-307, https://doi.org/10.1177/0022034516680585.

[14] C. Ganss, J. Klimek, N. Schwarz, A comparative profilometric in vitro study of the susceptibility of polished and natural human enamel and dentine surfaces to erosive demineralization, Arch. Oral Biol. 45 (2000) 897-902, https://doi.org/10.1016/s0003-9969(00)00041-8.

[15] C. Ganss, A. Lussi, I. Scharmann, T. Weigelt, M. Hardt, J. Klimek, N. Schlueter, Comparison of calcium analysis, longitudinal microradiography and profilometry for the quantitative assessment of erosion in dentine, Caries Res. 43 (2009) 422-429, https://doi.org/10.1159/000252975.

[16] A. Aden, P. Anderson, G.R. Burnett, R.J.M. Lynch, P.H. Tomlins, Longitudinal correlation of 3D OCT to detect early erosion in bovine enamel, Biomed. Opt. Express 8 (2017) 954-973, https://doi.org/10.1364/BOE.8.000954.

[17] C.H. Wilder-Smith, P. Wilder-Smith, H. Kawakami-Wong, J. Voronets, K. Osann and A. Lussi, Quantification of dental erosions in patients with GERD using optical coherence tomography before 
and after double-blind, randomized treatment with esomeprazole or placebo, Am. J. Gastroenterol. 104 (2009) 2788-2795, https://doi.org/10.1038/ajg.2009.441.

[18] M. Joshi, Techniques to evaluate dental erosion: a systematic review of literature, J. Clin, Diagnostic Res. (2016) 1-7, https://doi.org/10.7860/jcdr/2016/17996.8634.

[19] R.P. Shellis, C. Ganss, Y. Ren, D.T. Zero, A. Lussi, Methodology and models in erosion research: discussion and conclusions, Caries Res. 45 (2011) 69-77, https://doi.org/10.1159/000325971.

[20] A.B. Meireles, A.W. Vieira, L. Corpas, B. Vandenberghe, F.S. Bastos, P. Lambrechts, M.M. Campos, E.B. Las Casas, Dental wear estimation using a digital intra-oral optical scanner and an automated 3D computer vision method, Comput. Methods Biomech. Biomed. Engin. 19 (2016) 507514, https://doi.org/10.1080/10255842.2015.1043627.

[21] S. Kumar, A. Keeling, C. Osnes, D. Bartlett, S. O'Toole, The sensitivity of digital intraoral scanners at measuring early erosive wear, J. Dent. 81 (2019) 39-42, https://doi.org/10.1016/j.jdent.2018.12.005.

[22] S. Michou, C. Vannahme, A.R. Benetti, K.R. Ekstrand, 3D assessment of erosive tooth wear using intraoral scanner. An in vitro examination. [Abstract], 65th ORCA Congr. July 4-7, 2018, Copenhagen, Denmark, Caries Res., 2018, p. 470, https://doi.org/10.1159/000488302.

[23] V. Alaraudanjoki, H. Saarela, R. Pesonen, M.L. Laitala, H. Kiviahde, L. Tjäderhane, A. Lussi, P. Pesonen, V. Anttonen, Is a basic erosive wear examination (BEWE) reliable for recording erosive tooth wear on 3D models? J. Dent. 59 (2017) 26-32, https://doi.org/10.1016/j.jdent.2017.02.001.

[24] F. Marro, L. De Lat, L. Martens, W. Jacquet, P. Bottenberg, Monitoring the progression of erosive tooth wear (ETW) using BEWE index in casts and their 3D images: A retrospective longitudinal study, J. Dent. 73 (2018) 70-75, https://doi.

org/10.1016/j.jdent.2018.04.008.

[25] S. O'Toole, C. Osnes, D. Bartlett, A. Keeling, Investigation into the accuracy and measurement methods of sequential 3D dental scan alignment, Dent. Mater. 35 (2019) 495-500, https://doi.org/10.1016/j.dental.2019.01.012.

[26] K. Ahmed, J. Whitters, X. Ju, S. Pierce, C. MacLeod, C. Murray, Clinical monitoring of tooth wear progression in patients over a period of one year using CAD/CAM, Int. J. Prosthodont. 30 (2017) 153-155, https://doi.org/10.11607/ijp.4990.

[27] R.J.Y. Kim, J.M. Park, J.S. Shim, Accuracy of 9 intraoral scanners for complete-arch image acquisition: a qualitative and quantitative evaluation, J. Prosthet. Dent. 120 (2018) 895-903, https://doi.org/10.1016/j.prosdent.2018.01.035.

[28] A.S. Mennito, Z.P. Evans, J. Nash, C. Bocklet, A. L. Kelly, T. Bacro, M. Cayouette, M. Ludlow, W.G. Renne, Evaluation of the trueness and precision of complete arch digital impressions on a human maxilla using seven different intraoral digital impression systems and a laboratory scanner, J. Esthet. Restor. Dent. 31 (2019) 369-377, https://doi.org/10.1111/jerd.12485.

[29] H. Yu, F.J. Wegehaupt, A. Wiegand, M. Roos, T. Attin, W. Buchalla, Erosion and abrasion of tooth-colored restorative materials and human enamel, J. Dent. 37 (2009) 913-922, https://doi.org/10.1016/j.jdent.2009.07.006.

[30] S. Bell, Measurement good practice guide - a beginner's guide to uncertainty of measurement, (1999), https://doi.org/10.1111/j.1468-3148.2007.00360.x. 